\title{
La crianza que ejercen padres y madres con hijos adolescentes en el medio ruralk un estudio exploratorio
}

Rearing practices performed by mothers and fathers to adolescents from a rural area in the State of Mexico: an exploratory study

\author{
Hans Oudhof Van Barneveld \\ Norma Ivonne González Arratia López Fuentes \\ Sergio González Escobar \\ Erika Robles Estrada \\ Brenda Rodríguez Aguilar
}

\section{Resumen}

Se realizó un estudio exploratorio con el objetivo de identificar las prácticas de crianza que ejercen padres y madres de familia en una zona rural del Estado de México, para lo cual se utilizó una muestra no probabilística por cuotas de 30 padres y madres con hijos adolescentes. Asimismo, se aplicó un cuestionario con preguntas abiertas, diseñado para determinar los aspectos básicos de la crianza parental en el hogar. Los resultados indican que predomina una división del trabajo por género de tipo tradicionalista: las madres son las principales responsables del cuidado de los hijos. Las actividades primordiales que se realizan para la educación de los hijos son promover su formación escolar, fomentar la comunicación, inculcar valores, además de dar consejos y apoyo. Se encontró una gran variedad de cualidades que los padres se atribuyen a sí mismos en su papel de educadores, tales como ser comprensivos, exigentes, responsables y cariñosos. Las estrategias que se utilizan con mayor frecuencia en caso de desobediencia de los hijos son llamarles la atención, hablar con ellos, castigos no físicos y tratar de corregir su comportamiento. Se concluye que las prácticas de crianza que reportaron los participantes en el estudio no concuerdan con la imagen estereotipada que se tiene de la socialización familiar en el medio rural.

\section{Abstract}

An exploratory study was carried out with the purpose of identifying the rearing practices performed by mothers and fathers from a rural area in the State of Mexico. A non-probabilistic quota sample of 30 mothers and fathers with adolescent children was employed. A questionnaire, designed for determining the basic aspects of parental rearing, was administered. The results indicate that a traditionalist labor division by gender prevails; mothers have the main responsibility for taking care of their offspring. The primordial activities carried out for children's education are promoting their performance at school, communication, transmitting values, giving advice and support. A great variety of structures which parents attribute to themselves in their educating role was obtained, such as comprehensive, demanding, responsible and loving. The most frequently reported strategies for children's disobedience are scolding, talking with them, non-physical punishment and trying to correct their behavior. It is concluded that the rearing practices reported by the participants do not match the stereotyped image of family socialization in rural areas.

Palabras Clave: crianza, familia, rural, padres, madres.

Keywords: rearing, family, rural, fathers, mothers. 


\section{Introducción}

La crianza es una de las funciones más importantes que cumplen las familias en distintos contextos socioculturales; tiene como objetivo principal fomentar el sano crecimiento físico y psicológico de los hijos y la transmisión de valores, actitudes, creencias y pautas de comportamiento que son juzgados deseables de acuerdo con el entorno (Gracia \& Musitu, 2000; Musitu \& Cava, 2001; Palacios \& Rodrigo, 2001). Investigaciones transculturales sobre la vida familiar han mostrado que cuidar y apoyar a los hijos, promoviendo su desarrollo social adecuado y encaminándolos hacia una socialización apropiada, constituye una tarea universal de los padres, independientemente de las características del ambiente, época y estructura familiar. Al respecto, de acuerdo con los retratos familiares elaborados en un estudio realizado en treinta países, la crianza y el cuidado de los hijos surge como una de las funciones esenciales de la familia la cual se ha mantenido constante a través del tiempo (Poortinga \& Georgas, 2006).

Resulta importante señalar que el carácter universal de la crianza familiar no implica que las prácticas nombradas sean estáticas ni de carácter diacrónico. El proceso de cambio social y las transformaciones que se han generado en las mentalidades en, prácticamente, todas las culturas durante las últimas décadas, han originado modificaciones en la percepción sobre cómo debe llevarse a cabo la socialización en la actualidad y en las ideas acerca de cuáles son los modos de crianza adecuados. Uno de los cambios que se ha presentado en las últimas generaciones es la adopción de estilos de educación más permisivos por parte de muchos padres que fueron educados de manera eminentemente autoritaria (Ato, Galián \& Huéscar, 2007). Un ejemplo de ello es lo reportado en padres argentinos por Balzaho (2003), quien encontró que más de la mitad de ellos manifestó ejercer un estilo de crianza contrapuesto a las prácticas estrictas a las que fueron habituados. Esteinou (2008) señala que en las familias mexicanas contemporáneas los padres tienden a asumir formas más democráti- cas de disciplinar y educar a sus hijos que en épocas anteriores.

Las formas de crianza que se manejan en distintos sistemas familiares pueden variar entre culturas e incluso entre subculturas De acuerdo con Keller et al. (2006), las metas de socialización varían según las demandas del entorno, la estructura socioeconómica de la sociedad, la ecología humana o el tipo de comunidad, de ahí la creación de diferencias en las expectativas que los padres plantean para sus hijos, las cuales se reflejan en el tipo de valores, actitudes y cualidades de enseñanza que buscan transmitir. En reportes de investigación que dichos autores realizaron en madres de distintos países, se encontraron diferencias significativas en las concepciones de la crianza en comunidades con distintos modelos culturales. Un estudio de Suizo (2007) con padres de cuatro grupos étnicos en Estados Unidos, demostró que los padres de los grupos minoritarios en el estudio (asiático, latino y africano) preservan ciertas ideas de la crianza, asociadas a sus orígenes, particularmente algunas creencias tradicionales específicas, como el respetar a sus padres; aunque también adoptan prácticas y creencias de la cultura mayoritaria estadounidense. De esta manera, las metas que los padres conciben para sus hijos incluyen el tipo de valores y cualidades de vida que les gustaría que éstos tuvieran en la edad adulta, motivando y guiando las actividades diarias de la crianza.

Generalmente, se parte del estereotipo de la familia rural como "tradicional," caracterizada por un alto grado de interdependencia material y afectiva, la existencia de lazos familiares fuertes y rigidez en la distribución de roles entre padres, madres e hijos. Este tipo de familia es asociado con estrategias de crianza autoritarias, con un alto nivel de imposición y de control parental sobre los hijos, quienes tienen que adecuarse a las expectativas de los padres, y la existencia de una relación jerárquica y vertical entre generaciones. Los padres son muy exigentes con sus hijos, tienen un bajo grado 
de implicación con sus necesidades afectivas y dan pocas muestras de su aceptación como personas con deseos, intereses y opiniones propias. Esta visión se acerca al estilo parental autoritario descrito por Baumrind (1971) y Maccoby y Martin (1983), en el cual es considerada particularmente formal y distante la relación que tienen los hijos con la figura paterna.

Kağitçibaşi (2006) afirma que la familia tradicional se caracteriza por una interdependencia general (material y emocional) entre sus miembros, lo cual tiene implicaciones en la orientación de la crianza de los hijos al estimularse, principalmente, la obediencia en ellos. Se valoran altamente el respeto y las obligaciones familiares de los miembros, se regula la conducta a través de normas grupales y se otorga siempre prioridad a los intereses colectivos de la familia sobre el desarrollo individual de sus integrantes.

Los hallazgos de diversos estudios indican que este estereotipo de la familia rural tradicional ya no corresponde plenamente con la realidad que viven las familias contemporáneas en el campo. Muestra de ello fue un estudio comparativo sobre la vida familiar en una localidad urbana y una rural del Valle de Toluca en el estado de México realizado por Oudhof (1997); en él se encontró que en ambos lugares coexisten características "modernas" y "tradicionales" en las formas de organización familiar, pero se presentaron pocas diferencias significativas entre los hogares según lugar de residencia. Garduño y Cervantes (1995) investigaron las prácticas de crianza en 21 familias en la comunidad de Cholula, Puebla, donde los padres utilizan en mayor medida los consejos y el apoyo que el castigo. Flores, Cortés y Góngora (2008) encontraron muchas similitudes entre padres y madres rurales y urbanos en el estado de Yucatán en lo que se refiere a la percepción de cómo debe ejercerse la crianza, en ambos casos, destacaron la interacción y comunicación familiar, el apoyo a los hijos y también la disciplina; pero sin llegar a los golpes.
En una encuesta del Sistema Nacional para el Desarrollo Integral de la Familia (2005, SNDIF) sobre la dinámica familiar que prevalece en el país, se señala que existen algunas tendencias hacia mayor conservadurismo en las familias rurales, particularmente en cuanto a los patrones de relación intergeneracional e intergenérico entre sus miembros y acerca de la responsabilidad que tienen las mujeres en el hogar, aunque en otros aspectos, las diferencias con las familias urbanas son muy reducidas o nulas.

El presente estudio es de carácter exploratorio; su objetivo fue identificar las creencias y prácticas de crianza en padres y madres de hijos adolescentes en una zona rural del estado de México. Forma parte de una investigación más amplia en la cual participan grupos de investigación de varias universidades mexicanas en distintas zonas del país; el objetivo de dicha investigación es desarrollar y validar una escala para medir la crianza que ejercen padres y madres de familia con hijos adolescentes y de esta manera contar con un instrumento válido y confiable a nivel nacional, para ir más allá de contextos regionales específicos. Los resultados que se reportan en este trabajo corresponden, únicamente, a la primera fase de este proyecto, cuya finalidad es obtener indicadores más claros y precisos para poder medir el ejercicio de la crianza en padres de familia y la percepción de los hijos al respecto.

\section{Método}

\section{Participantes}

El estudio se llevó a cabo en comunidades rurales en la zona occidental del estado de México; las localidades rurales fueron definidas como aquellas caracterizadas por una baja densidad poblacional, una importante presencia de las actividades agropecuarias en la estructura económica y la ausencia (parcial) de la infraestructura y servicios públicos que son propios de las aglomeraciones urbanas. Se tra- 
bajó con una muestra no probabilística por cuotas de 30 madres y 30 padres con 30 hijos y 30 hijas adolescentes en la edad de 12 a 17 años. El rango de edad de los padres fue de 32 a 63 años y el número de hijos fue de 2 a 6 . El $50 \%$ de los padres cuenta con el nivel educativo de secundaria y la proporción que tiene estudios de primaria y preparatoria fue de $20 \%$ para ambos casos. La edad de las madres oscila entre los 27 y 53 años, con 1 a 6 hijos; predominan entre ellas los niveles educativos de primaria $(33 \%)$, secundaria (33\%) y preparatoria $(20 \%)$.

\section{Instrumento}

Se diseñó un cuestionario con ocho preguntas abiertas que abordan aspectos básicos de la crianza: la forma de educar a los hijos/ as; la autopercepción que se tiene como padre/ madre; cómo se demuestra afecto a los hijos/ hijas; y las cosas que hacen cuando los hijos/ as desobedecen. El instrumento fue elaborado de manera colectiva, con base en experiencias en investigaciones previas sobre crianza, realizadas por los integrantes del proyecto "Crianza, personalidad y adolescencia" desarrollado por distintos cuerpos académicos de diversas universidades públicas, pertenecientes al Consorcio de Universidades Mexicanas; asimismo, corresponde a la primera etapa (exploratoria) de la investigación. Las preguntas se dirigen exclusivamente a las actividades que padres y madres llevan a cabo en la crianza de sus hijos adolescentes.

\section{Procedimiento}

La aplicación se realizó de manera directa en las viviendas de los participantes, bajo los principios de colaboración voluntaria y anónima y garantizando la confidencialidad de los datos proporcionados.

\section{Análisis de datos}

Para el análisis de la información se empleó un análisis de contenido; las respuestas fueron codificadas y posteriormente clasificadas, de tal manera que afirmaciones similares de los participantes fueron agrupadas en un mismo rubro. Se utilizó la estadística descriptiva; se calcularon frecuencias y porcentajes de la ocupación de los padres, las prácticas que se utilizan para educar a los hijos, la autopercepción como padre o madre, las formas de mostrar afecto a los hijos y el manejo de la desobediencia.

\section{Resultados}

A continuación se describen los principales resultados obtenidos en el estudio. Cabe aclarar que, salvo en el caso de la ocupación de los participantes, se podría dar más de una respuesta en las demás preguntas, por lo que la suma de los porcentajes rebasa el 100\%.

En la tabla 1 se presentan las ocupaciones de los participantes de la muestra. En el caso de los padres de familia, todos trabajan fuera del hogar, realizando sus labores principalmente como campesinos (30\%), comerciantes (23\%), albañiles (13\%) o mecánicos (7\%). En lo que se refiere a las participantes, la gran mayoría de ellas, el $76 \%$ del total, se dedica exclusivamente al hogar, pero algunas trabajan como comerciantes o maestras.

Con respecto a la pregunta sobre lo que hacen los padres para criar a los hijos, la principal actividad mencionada se refiere a procurar que acudan a la escuela, en el $37 \%$ del total de los casos, seguida por la comunicación (33\%), enseñar valores (20\%), dar consejos (18\%) y dar ayuda y apoyo (17\%), como se observa en la tabla 2. Los padres dan mayor énfasis al aspecto de la comunicación y la ayuda, mientras que las madres dan mayor prioridad a inculcar 
valores y a la orientación. Cabe observar que las actividades de crianza dirigidas a los hijos y las hijas fueron muy similares; incluso hubo participantes que mencionaron de manera explícita que no hacen una diferenciación en el trato de los hijos según el género.

En lo que se refiere a la autopercepción de los padres y las madres, se encontró una gran variedad de apreciaciones acerca de cómo se definen a sí mismos en su calidad de educadores, por lo que se reportan, únicamente, las respuestas con mayores frecuencias (superiores al 10\%). Predominaron los calificativos de tipo afectivo y relacionados con el apoyo a los hijos, tales como "comprensivo", "responsable" y "cariñoso", pero también hubo varios participantes que se juzgaron como "estrictos" y "exi- gentes". Algunos valoraron su desempeño parental como "normal", "más o menos" o "regular"; incluso, en algunos casos señalaron que aún les falta mucho por aprender como padre o madre (tabla 3).

Para mostrar afecto a los hijos, no se encontró un patrón claro en las formas que se especificaron; en la tabla 4 se observa que ninguna categoría representa proporciones mayores a $20 \%$ de la muestra total, además, se combinan aspectos verbales (platicar, decir que se quiere a los hijos), de presencia (estar con ellos), expresiones físicas (abrazos, besos, caricias) y muestras de apoyo. Los padres muestran una ligera tendencia a priorizar, en mayor medida, estar presente cuando los hijos lo necesiten, mientras que las madres dieron prioridad a las expresiones físicas de afecto.

Tabla 1.

Ocupación de los participantes

\begin{tabular}{lcc}
\hline Ocupación & $\begin{array}{c}\text { Padres (\%) } \\
\mathrm{n}=30\end{array}$ & $\begin{array}{c}\text { Madres (\%) } \\
\mathrm{n}=30\end{array}$ \\
\hline Hogar & - & 76 \\
Campesino & 30 & - \\
Comerciante & 23 & 10 \\
Albañil & 13 & - \\
Mecánico & 7 & - \\
Maestra & - & 7 \\
Otro & 27 & 7 \\
\hline
\end{tabular}

\section{Tabla 2.}

Prácticas más frecuentes para educar a los hijos/hijas

\begin{tabular}{lccc}
\hline Actividades & $\begin{array}{c}\text { Total (\%) } \\
\mathrm{N}=60\end{array}$ & $\begin{array}{c}\text { Padres (\%) } \\
\mathrm{n}=30\end{array}$ & $\begin{array}{c}\text { Madres (\%) } \\
\mathrm{n}=30\end{array}$ \\
\hline Mandarlos a la escuela & 37 & 37 & 37 \\
Comunicación/hablar & 33 & 40 & 27 \\
Enseñar/inculcar valores & 20 & 13 & 27 \\
Dar consejos & 18 & 17 & 20 \\
Dar ayuda/apoyo & 17 & 20 & 13 \\
Poner el ejemplo & 5 & 10 & - \\
Orientación & 5 & - & 10 \\
Corregir & 5 & 7 & 3 \\
\hline
\end{tabular}




\section{Tabla 3.}

Autopercepción como padre/madre

\begin{tabular}{lccc}
\hline Características & $\begin{array}{c}\text { Total (\%) } \\
\mathrm{N}=60\end{array}$ & $\begin{array}{c}\text { Padres (\%) } \\
\mathrm{n}=30\end{array}$ & $\begin{array}{c}\text { Madres (\%) } \\
\mathrm{n}=30\end{array}$ \\
\hline Comprensivo & 31 & 27 & 37 \\
Estricto/exigente & 22 & 23 & 20 \\
Responsable & 15 & 20 & 10 \\
Cariñoso & 15 & 13 & 17 \\
$\begin{array}{l}\text { Normal/regular/me falta } \\
\text { aprender }\end{array}$ & 13 & 13 & 13
\end{tabular}

\section{Tabla 4.}

Formas de mostrar afecto a los hijos/hijas

\begin{tabular}{lccc}
\hline Actividades & $\begin{array}{c}\text { Total }(\%) \\
\mathrm{N}=60\end{array}$ & $\begin{array}{c}\text { Padres (\%) } \\
\mathrm{n}=30\end{array}$ & $\begin{array}{c}\text { Madres (\%) } \\
\mathrm{n}=30\end{array}$ \\
\hline Hablar/platicar & 20 & 20 & 20 \\
Estar con el(la) & 17 & 27 & 7 \\
Abrazos/besos/caricias & 15 & 10 & 20 \\
Darle cariño, amor, afecto & 13 & 17 & 10 \\
Ayudar/apoyar & 13 & 13 & 13 \\
Decir que lo/la quiero & 12 & 7 & 17 \\
\hline
\end{tabular}

En caso de desobediencia de los hijos e hijas, los participantes optan en mayor medida por regañar y llamar la atención (38\%) y por hablar con los hijos y tratar de hacerles entender que su comportamiento no es correcto (37\%). También expresaron utilizar con cierta frecuencia la aplicación de castigos, en principio, de tipo económico (no darles dinero), no dejar salir a los hijos o privarles temporalmente de algo importante, pero no se recurre al recurso de los golpes 0 a las sanciones físicas. En la tabla 5 se observa que las madres regañan y castigan más que los padres, quienes prefieren más frecuentemente el diálogo.

Tabla 5.

Manejo de la desobediencia de los hijos/hijas

\begin{tabular}{lccc}
\hline Actividades & $\begin{array}{c}\text { Total (\%) } \\
\mathrm{N}=60\end{array}$ & $\begin{array}{c}\text { Padres (\%) } \\
\mathrm{n}=30\end{array}$ & $\begin{array}{c}\text { Madres (\%) } \\
\mathrm{n}=30\end{array}$ \\
\hline Regañar/llamarle la atención & 38 & 33 & 43 \\
Platicar/hablar & 37 & 43 & 30 \\
Castigo & 35 & 27 & 43 \\
Tratar de corregir & 12 & 17 & 7 \\
\hline
\end{tabular}




\section{Discusión}

De acuerdo con las ocupaciones de los participantes en el estudio, se puede inferir que en la mayoría de los hogares rurales del estudio sigue predominando una división interna del trabajo de tipo tradicionalista: el rol básico del padre es de proveedor, mientras que la madre es la principal encargada de la crianza, el cuidado de los hijos y las labores domésticas. En este aspecto de la organización familiar, se confirma la vigencia del estereotipo de género, según el cual son las mujeres las que asumen más cargas y responsabilidades familiares (Monreal \& Martínez, 2010). Esto coincide con los resultados de la Encuesta nacional sobre la dinámica familiar realizada por el SNDIF (2005), en la cual se encontró que en el medio rural se acentúa más la prevalencia de una noción conservadora acerca del papel de la mujer en la convivencia familiar, al considerar que es la principal responsable de mantener a la familia unida por lo cual no es deseable que las mujeres trabajen fuera de la casa si tienen hijos pequeños.

En las prácticas de crianza que se ejercen en el hogar sobresale la prioridad que otorgan los padres al papel de la escuela para el aprendizaje de los hijos, de tal forma se confirma que fomentar la educación escolar es un objetivo socializador primordial para ellos, a partir de la idea generalizada según la cual la preparación académica constituye una herramienta muy importante para el futuro de los hijos, para que salgan adelante (Flores et al., 2008; González \& González, 1989). Adicionalmente, se enfatiza el aspecto afectivo de la relación con los hijos, a través de la comunicación y el apoyo, así como la orientación mediante los consejos y la transmisión de valores. Actividades como corregir y llamar la atención, que pertenecen a la dimensión de control y poner límites de la crianza, fueron mencionadas con menor frecuencia. Es posible que lo anterior se relacione con la etapa de desarrollo en la que se encuentran los hijos, la cual conlleva con frecuencia un ajuste en el estilo de crianza de los padres (Musitu \& Cava,
2001). Arnett (2008) menciona que es común observar una mayor flexibilización en la socialización parental a medida que crecen los hijos, y entran en a la etapa de la adolescencia. Asimismo, diversos autores señalan que la crianza actualmente tiende a ser más democrática y menos autoritaria que antes (Ato, Galián \& Huéscar, 2007; Esteinou, 2008).

Llama la atención que se observó un alto grado de similitud en la caracterización de la crianza que dieron los participantes según género: no se detectaron diferencias claras entre padres y madres ni en la forma de educar a los hijos y las hijas. Estos hallazgos reflejan ciertas modificaciones que se han presentado en los roles y las pautas de comportamiento en décadas recientes, de acuerdo con lo que afirman Rocha y Díaz-Loving (2011): las cosas han cambiado para muchos hombres y mujeres, se ha originado una gran diversidad tanto en los hijos y las hijas como en las madres y los padres.

La autopercepción de los participantes en cuanto a su desempeño en el rol parental arrojó un alto grado de variación en las caracterizaciones que se atribuyen a sí mismos. Se infiere que para los participantes en el estudio no hay un modelo dominante sobre cómo es o debe ser, en su caso, un buen padre y una buena madre. Algo similar se encontró en las formas de mostrar afecto; se mencionó una amplia pluralidad de expresiones físicas, verbales, muestras de apoyo y manifestaciones sobre la importancia que representan los hijos. En estos aspectos los datos de este estudio no permiten hacer generalizaciones ni establecer diferencias claras entre madres y padres, por lo que se considera necesaria mayor investigación al respecto.

En lo que se refiere a las formas de manejar la desobediencia de los hijos, se obtuvo que se utilizan tres estrategias parentales en similar proporción: regañar y llamar la atención, platicar con los hijos y recurrir a castigos no físicos. En la comparación por sexo, se encontró que 
las madres se inclinan más por el regaño y el castigo, lo que se debe posiblemente a su mayor cercanía con los hijos, ya que la mayoría se dedica exclusivamente a la crianza y al hogar, por lo cual están más al pendiente del comportamiento de los hijos. En cambio, los padres expresaron una mayor preferencia por el diálogo.

Si bien estos hallazgos son una contribución a la mayor comprensión de las prácticas de crianza que se ejercen en zonas rurales mexicanas, es importante señalar algunas limitantes del estudio. En primer lugar, la investigación realizada fue de carácter exploratorio, en ella se utilizó un cuestionario con preguntas abiertas y generales, como una primera aproximación al objeto de estudio. Sin embargo, se requiere más precisión y mayor análisis en la información obtenida respecto a algunos de los aspectos abordados, particularmente la percepción que tienen los padres de su propio rol parental y las formas de mostrar afecto a los hijos. En estas dimensiones, los datos proporcionados fueron muy heterogéneos y dispersos, de modo que no fue posible obtener una categorización conceptualmente clara; se sugiere abordar estas cuestiones a partir de instrumentos de medición con reactivos más estructurados y específicos en investigaciones futuras. En segundo lugar, al tratarse de una muestra relativamente pequeña en una zona específica del estado de México, no es posible generalizar los resultados.

Se concluye que en las prácticas de crianza que reportan las madres y los padres del estudio se da mucha importancia a la dimensión afectiva de la crianza a través del apoyo y el cariño, elemento que se descubrió igual o incluso más importante que la dimensión de control y disciplina. Esto no concuerda con la imagen estereotipada de la socialización familiar tradicional en el medio rural, por lo cual se considera conveniente realizar más estudios sobre las prácticas de crianza en este entorno.
Arnett, J.J. (2008). Adolescencia y adultez emergente. Un enfoque cultural. México: Pearson Prentice Hall.

Ato, E., Galián, M., \& Huéscar, E. (2007). Relaciones entre estilos educativos, temperamento y ajuste en la infancia: Una revisión. Anales de psicología, 23 (1), 33-40.

Balzaho, S. (2003). No todo tiempo pasado fue mejor... Percepciones de las diferencias generacionales en la crianza y educación de los hijos. Estudios sobre las culturas contemporáneas, 9 (18), 103-126.

Baumrind, D. (1971). Current patterns of parental authority. Developmental psychology Monograph, 4.

Esteinou, R. (2008). La familia nuclear en México: Lecturas de su modernidad. Siglo XVI al XX. México: CIESAS/Miguel Ángel Porrúa.

Flores, M.M., Cortés, M.L., \& Góngora, E.A. (2008). Familia, crianza y personalidad: Una perspectiva etnopsicológica. Mérida: Universidad Autónoma de Yucatán.

Garduño, L.R., \& Cervantes, D. (1995). Prácticas de crianza en familias participantes en un proyecto de desarrollo comunitario en el Estado de Puebla. Revista Latinoamericana de Estudios Educativos, 25 (3), 87-108.

González y González, I. (1989). La índole de los mexicanos vista por ellos mismos. Nexos, 114, 31-44.

Gracia, E., \& Musitu, G. (2000). Psicología social de la familia. Barcelona: Paidós.

Kağitçibaşi, C. (2006). Theoretical perspectives of family change. En J. Georgas, J.W. Berry, F.J.R. van de Vijver, C. Kağitçibassi \& Y.H. Poortinga (Eds.), Families across cultures. A 30-nation psychological study (pp. 72-89). Cambridge: University Press.

Keller, H., Lamm, B., Abels, M., Yovsi, R., Borke, J., Jensen, H., Papaligoura, Z., Holub, C., Lo, W., Tomiyama, A.J., Su, Y., Wang, Y., \& Chaudhary, N. (2006). Cultural models, socialization goals and parenting ethnotheories: A multicultural analysis. Journal of CrossCultural Psychology, 37 (2), 155-172.

Maccoby, E.E., \& Martin, J.A. (1983). Socialization in the context of the family: Parent-child interaction. En E.M. Hetherington (Ed.), Socialization, personality and social development. Volume IV (pp. 1-101). New York: John Wiley.

Monreal, M.C. \& Martínez, B. (2010). Esquemas de género y desigualdades sociales. En L.V. Amador \& M.C. Monreal (Eds.), Intervención social y género (pp. 73-94). Madrid: Narcea.

Musitu, G., \& Cava, M.L. (2001). La familia y la educación. Barcelona: Octaedro

Oudhof, H. (1997). Modernización, condiciones regionales y vida familiar en el Valle de Toluca. Cuaderno de investigación. Toluca: Universidad Autónoma del Estado de México.

Palacios, J., \& Rodrigo M. (2001). La familia como contexto de desarrollo humano. En J. Palacios \& M. Rodrigo (Eds.), Familia y desarrollo humano (pp. 25-44). Madrid: Alianza.

Poortinga, Y.H., \& Georgas, J. (2006). Family portraits from 30 countries: An overview. En J. Georgas, J.W. Berry, F.J.R. van de Vijver, C. Kağitçibaşi \& Y.H. Poortinga (Eds.), Families across cultures. A 30-nation psychological study (pp. 90-99). Cambridge: University Press.

Rocha, T.E., \& Díaz-Loving, R. (2011). Identidades de género. Más allá de cuerpos y mitos. México: Trillas.

Sistema Nacional para el Desarrollo Integral de la Familia (2005). Encuesta nacional sobre la dinámica de las familias, 2005: Informe. México: DIF.

Suizzo, M.A. (2007). Parents' goals and values for children: Dimensions of independence across four U.S. ethnic groups. Journal of CrossCultural Psychology, 38 (4), 506-530. 